\title{
Determination of the $s$-wave pion-nucleon threshold scattering parameters from the results of experiments on pionic hydrogen
}

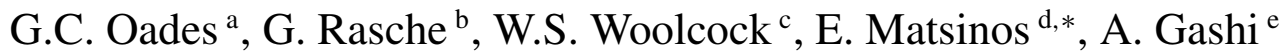 \\ ${ }^{a}$ Institute of Physics and Astronomy, Aarhus University, DK-8000 Aarhus C, Denmark \\ b Institut für Theoretische Physik der Universität, Winterthurerstrasse 190, CH-8057 Zürich, Switzerland \\ c Department of Theoretical Physics, IAS, The Australian National University, Canberra, ACT 0200, Australia \\ ${ }^{\mathrm{d}}$ Varian Medical Systems Imaging Laboratory GmbH, Täfernstrasse 7, CH-5405 Baden-Dättwil, Switzerland \\ e Mediscope AG, Alfred Escher-Str. 27, CH-8002 Zürich, Switzerland
}

Received 23 February 2007; received in revised form 26 July 2007; accepted 26 July 2007

Available online 14 August 2007

\begin{abstract}
We give the conversion equations which lead from experimental values of the $3 p \rightarrow 1 s$ transition energy in pionic hydrogen and the total width of the $1 s$ level to values of the $s$-wave threshold scattering parameters for the processes $\pi^{-} p \rightarrow \pi^{-} p$ and $\pi^{-} p \rightarrow \pi^{0} n$ respectively. Using a three-channel potential model, we then calculate the electromagnetic corrections to these quantities, which remove the effects of the Coulomb interaction, the external mass differences and the presence of the $\gamma n$ channel. We give the $s$-wave scattering parameters obtained from the present experimental data and these electromagnetic corrections. Finally we discuss the implications for isospin invariance.
\end{abstract}

(c) 2007 Elsevier B.V. All rights reserved.

PACS: $13.40 . \mathrm{Ks} ; 13.75 . \mathrm{Gx} ; 36.10 . \mathrm{Gv}$

Keywords: Pionic hydrogen; $\pi N$ electromagnetic corrections

\footnotetext{
* Corresponding author. Tel.: +41 56 2030460; fax: +41 562030405.

E-mail address: evangelos.matsinos@varian.com (E. Matsinos).
} 


\section{Introduction}

Accurate values of the $s$-wave scattering parameters $a_{c c}$ and $a_{c 0}$, for the processes $\pi^{-} p \rightarrow$ $\pi^{-} p$ and $\pi^{-} p \rightarrow \pi^{0} n$ at the $\pi^{-} p$ threshold, can be obtained from measurements of an $n p \rightarrow$ $1 s$ transition energy and the width of the $1 s$ level in pionic hydrogen $(\mathrm{H})$ via a refinement of the DGBT formulae [1]. As the most accurate experimental data available are for the $3 p \rightarrow 1 s$ transition energy, we will consider only this case in the present paper. The most recent fully published experimental values of these quantities are given in Ref. [2]. A successor experiment [3] is nearing completion, and preliminary results from this experiment for the transition energy [4] and for the width [5] are available. In Section 2 of this paper we shall give a detailed derivation of the relevant conversion equations which connect the experimental data with the scattering parameters, paying particular attention to the sources of uncertainty and making clear which pion-nucleon $(\pi N)$ interactions contribute to the parameters. We use these conversion equations to obtain the values of $a_{c c}$ and $a_{c 0}$ from the results in Refs. [4,5]. These equations can also be used for the final results of the experiment [3] and for those of any future experiment.

The conversion equations just mentioned are based on the formalism of Ref. [6]. It was the first work to generalise the systematic derivation of the DGBT formulae for a one-channel system $[7,8]$ to a multichannel system, and to apply the results to pionic $\mathrm{H}$. This systematic derivation is based on the general principles of scattering theory and on the analytic continuation of the matrix $\mathbf{K}$ (defined later in Eq. (3)) into the complex $s$-plane, to those (complex) values of $s$ which correspond to the levels of pionic $\mathrm{H}$. All this is independent of any phenomenological model of the dynamics of the system. The formalism of Ref. [6] enables one to extract directly and unambiguously from the experimental data the values of precisely defined threshold scattering parameters $a_{c c}$ and $a_{c 0}$, whose errors are dominated by the experimental errors of these atomic data. Then, as a second quite distinct step, a dynamical calculation of the electromagnetic (EM) corrections to these quantities is needed if we are to obtain hadronic scattering parameters.

In Section 3 we shall describe our calculation of the electromagnetic (EM) corrections to the threshold parameters, which uses a three-channel potential model to remove the effects of the Coulomb interaction, the external mass differences and the presence of the $\gamma n$ channel. We shall also give the values, together with estimated errors, of the corrected scattering parameters (denoted by $\tilde{a}_{c c}$ and $\tilde{a}_{c 0}$ ) which result from $a_{c c}$ and $a_{c 0}$. In Section 4 we shall set our calculation of the EM corrections in the context of previously published calculations, and attempt to summarise our present understanding of these corrections. We shall see that, while the experimental data from pionic $\mathrm{H}$ yield extremely accurate values of $a_{c c}$ and $a_{c 0}$, the calculation of the EM corrections needed to obtain hadronic quantities is subject to a great deal of uncertainty.

In Section 5 we shall use the value of the $s$-wave scattering length $\tilde{a}_{+}$for $\pi^{+} p$ elastic scattering, obtained from a recent phase-shift analysis (PSA) [9] of the data up to a laboratory pion kinetic energy $\left(T_{\pi}\right)$ of $100 \mathrm{MeV}$, which employed the EM corrections of Ref. [10], also calculated using a potential model. To ensure that the two calculations of EM corrections were consistent, we adopted, for the hadronic potentials in the calculation described in Section 3, the same forms as were used for the corrections given in Ref. [10]. We shall show that this value of $\tilde{a}_{+}$shows a large and significant difference from the value of $\tilde{a}_{c c}+\sqrt{2} \tilde{a}_{c 0}$, calculated from the most recent values of $\tilde{a}_{c c}$ and $\tilde{a}_{c 0}$ given in Section 3 .

This reinforces the evidence for the violation of isospin invariance given in Ref. [9], from the inability of the phase shifts, obtained from the analysis of $\pi^{ \pm} p$ elastic scattering data alone, to reproduce the data on $\pi^{-} p$ charge exchange scattering. As discussed in Ref. [9], at least part of this violation of isospin invariance has to be attributed to the presence of residual EM effects 
in the phase shifts obtained from the experimental data after the application of our corrections. This is because the EM corrections calculated using a potential model are only partial (stage 1) corrections; further (stage 2) corrections are needed to remove these residual EM effects.

We do not include in the present paper the information from the accurate measurement of a transition energy in pionic deuterium (D) [11]. There is an extensive literature on this subject [12-14]. The most recent of these papers [14] concludes tentatively that, when stage 2 corrections (calculated using chiral perturbation theory at leading order) are taken into account, the atomic data from pionic $\mathrm{H}$ and pionic $\mathrm{D}$ taken by themselves satisfy isospin invariance at the hadronic level. However, Ref. [14] emphasises that an improved measurement of a transition energy in pionic $\mathrm{D}$ is needed, as is a major effort on the theoretical side, to extract a scattering parameter with better precision. This complex subject lies outside the scope of this paper. Our aim here is to present what can be learned directly from the experimental data for pionic $\mathrm{H}$ and to relate the results consistently to those obtained from our PSA [9].

\section{Conversion formulae}

The first piece of experimental data from pionic $\mathrm{H}$ is the $3 p \rightarrow 1 s$ transition energy $W(3 p \rightarrow$ $1 s)$. The positions of the $3 p$ and $1 s$ levels relative to the $\pi^{-} p$ threshold we call $W(3 p)$ and $W(1 s)$ respectively. For the numerical calculations we will use always the most recent values of the physical constants [15]; errors will be given only if they are relevant for our purpose.

Non-relativistically the point charge Coulomb potential $V^{\mathrm{pc}}$ contributes -359.438 eV to $W(3 p)$. The shift due to vacuum polarisation is $-0.011 \mathrm{eV}$ [16]. Furthermore the $3 p$ level is split by the hyperfine interaction and the relativistic effects. The former is discussed on page 413 of Ref. [6], while the latter is given by Eqs. (9), (10) of Ref. [17]. For $j=3 / 2$ these two effects add to $-0.0013 \mathrm{eV}$, for $j=1 / 2$ to $-0.0055 \mathrm{eV}$. With the present experimental resolution of $W(3 p \rightarrow 1 s)$ this splitting cannot be seen. We therefore take a weighted average of $-0.003 \mathrm{eV}$, with an uncertainty of $0.002 \mathrm{eV}$, as the contribution of these effects to $W(3 p)$. The shift in the $3 p$ level due to the hadronic interaction, including the effect of the extended charge distributions of $\pi^{-}$and $p$, is negligible. Thus

$$
W(3 p)=-359.452(2) \mathrm{eV} \text {. }
$$

Since in the transition $3 p \rightarrow 1 s$ the kinetic recoil energy of the $\pi^{-}$atom in the c.m. frame is $0.004 \mathrm{eV}$, we have

$$
W(1 s)=-W(3 p \rightarrow 1 s)-359.456(2) \mathrm{eV} .
$$

Non-relativistically $V^{\mathrm{pc}}$ contributes $-3234.945 \mathrm{eV}$ to $W(1 s)$. The relativistic shift for an infinitely heavy proton is $-0.215 \mathrm{eV}$. This comes either from Ref. [17] or from integration of the Klein-Gordon equation [16]. In addition, both these references give a relativistic recoil shift of $+0.037 \mathrm{eV}$ and a contribution of $+0.010 \mathrm{eV}$ due to the magnetic moment of the proton. Ref. [16] also quotes a contribution of $-0.018 \mathrm{eV}$ for vacuum polarisation at $O\left(\alpha^{3}\right)$ and a vertex correction of $+0.007 \mathrm{eV}$. The potential $V^{\mathrm{vp}}$ for vacuum polarisation at $O\left(\alpha^{2}\right)$ is the Uehling potential, modified at short distances due to the finite extension of the charge distributions of $\pi^{-}$ and $p$. Adding $V^{\mathrm{vp}}$ to $V^{\mathrm{pc}}$, our calculations give a shift of $-3.244 \mathrm{eV}$. The finite extension of the charge distributions also modifies $V^{\mathrm{pc}}$ itself through the addition of a potential

$$
V^{\mathrm{ext}}=-\alpha / r(\operatorname{erf}(r / c)-1), \quad \text { with } c^{2}=\frac{2}{3}\left(\left\langle r^{2}\right\rangle_{\pi}+\left\langle r^{2}\right\rangle_{p}\right)
$$


The rms charge radii of $\pi^{-}$and $p$ were taken from Refs. $[18,19]$. The addition of $V^{\text {ext }}$ to $V^{\text {pc }}$ gives a further shift of $+0.100 \mathrm{eV}$. The $1 s$ position arising from the purely electromagnetic contributions considered so far is $-3238.268 \mathrm{eV}$. Nearly all the difference between this value and the widely used "total electromagnetic binding energy" in Table 1 of Ref. [16] comes from the change in the value of $-\frac{1}{2} \alpha^{2} m_{c}$, due to the use of the most recent value [15] of the mass $\mu_{c}$ of the $\pi^{-}$.

There are sizeable contributions to $W(1 s)$ from the interference of $V^{\mathrm{vp}}$ and $V^{\text {ext }}$ with the hadronic interaction $\tilde{V}$ (see Section $4 ; \tilde{V}$ as well as the other potentials are matrices in a coupledchannel calculation). We determined this shift, as well as the width, directly in a subthreshold calculation with mass differences included. To this end we evaluated the shift and the width due to $\tilde{V}$, for the two cases $V^{\mathrm{pc}}+V^{\mathrm{vp}}+V^{\mathrm{ext}}+\tilde{V}$ and $V^{\mathrm{pc}}+\tilde{V}$. It turned out that, when the direct contributions of $V^{\mathrm{vp}}$ and $V^{\mathrm{ext}}(-3.244 \mathrm{eV}$ and $+0.100 \mathrm{eV}$ respectively) are accounted for, the shift due to the interference of $\left(V^{\mathrm{vp}}+V^{\mathrm{ext}}\right)$ with $\tilde{V}$ is numerically negligible. The error, estimated by varying the range of $\tilde{V}$, is $0.003 \mathrm{eV}$. The cancellation of the two interference contributions due to $V^{\mathrm{vp}}$ and $V^{\text {ext }}$ was already noted implicitly in Ref. [16]. The result for the width will be given later.

The shift of the purely electromagnetic $1 s$ position given above, due to the interference of $\tilde{V}$ with $V^{\mathrm{pc}}$ only, we call $\Delta W$. Thus, from the result in the preceding paragraph, we have

$$
W(1 s)=-3238.268(8) \mathrm{eV}+\Delta W,
$$

and, using Eq. (1),

$$
\Delta W=-W(3 p \rightarrow 1 s)+2878.812(8) \mathrm{eV} .
$$

Note that, for practical purposes, the shift $\Delta W$ defined in this way is equal to the quantity $\epsilon_{1 s}$ defined in Refs. $[2,4,16]$. This is because, though we have taken account of the short-range effects fully, by including the interference of $V^{\mathrm{pc}}$ with $\left(V^{\mathrm{vp}}+V^{\mathrm{ext}}\right)$ in the calculation of the shift and width (rather than as a correction to the scattering parameters), the effect is negligible in the case of the shift. In the last two equations we have given the uncertainty explicitly. It is dominated by the uncertainty in $\mu_{c}$.

The hadronic interaction couples the $\pi^{-} p$ channel to the $\pi^{0} n$ and $\gamma n$ channels. As well as creating the shift $\Delta W$, it also makes the $1 s$ level unstable, with a width $\Gamma$. Compared to $\Gamma$, the hadronic and electromagnetic widths of the $3 p$ level can be neglected numerically. The process then is a three-body decay of the well defined $3 p$ level, going via the unstable $1 s$ level: $3 p \rightarrow$ $\left(\pi^{0} n\right) \gamma$ or $(\gamma n) \gamma$. The parentheses indicate that $\left(\pi^{0} n\right)$ and $(\gamma n)$ are the decay products of the intermediate $1 s$ level.

The treatment of such a three-body decay mechanism is given in detail in Section 4 of Ref. [20]. Let $s$ be the square of the invariant mass of $\left(\pi^{0} n\right)$ (or $\left.(\gamma n)\right)$. It turns out that the decay amplitude is proportional to

$$
\left(s-M_{0}^{2}+i M_{0} \Gamma\right)^{-1} \text {. }
$$

Here $M_{0}=\mu_{c}+m_{p}+W(1 s), m_{p}$ being the proton mass. We have neglected a possible dependence of $\Gamma$ on $s$. Using the relation between $s$ and the energy $E$ of the photon in the rest frame of the decaying $3 p$ state, and noting that $W(3 p \rightarrow 1 s) \ll M_{0}$, one sees that the spectrum of $E$ becomes proportional to

$$
\left[(E-W(3 p \rightarrow 1 s))^{2}+\frac{1}{4} \Gamma^{2}\right]^{-1} .
$$


Thus the measurement of this spectrum gives $W(3 p \rightarrow 1 s)$ and $\Gamma$.

We now derive the conversion equations which give the values of the elements $a_{c c}$ and $a_{c 0}$ of a, the $s$-wave scattering matrix for the $\left(\pi^{-} p, \pi^{0} n, \gamma n\right)$ system at the $\pi^{-} p$ threshold, in terms of $W(3 p \rightarrow 1 s)$ and $\Gamma$. We use $c, 0$ and $\gamma$ to denote the three coupled channels and $q_{c}, q_{0}$ and $k$ to denote the channel momenta in the c.m. frame. The matrix $\mathbf{a}$ is the value of $\mathbf{K}$ at the $\pi^{-} p$ threshold $q_{c}=0 . \mathbf{K}$ is defined in terms of the unitary matrix $\mathbf{S}$ by

$$
\begin{aligned}
& \mathbf{t}=-i\left(\mathbf{S}-\mathbf{1}_{3}\right)\left(\mathbf{S}+\mathbf{1}_{3}\right)^{-1}, \\
& \mathbf{K}^{-1}=\left(\begin{array}{ccc}
C_{0}(\eta) & 0 & 0 \\
0 & 1 & 0 \\
0 & 0 & 1
\end{array}\right) \mathbf{Q}^{1 / 2} \mathbf{t}^{-1} \mathbf{Q}^{1 / 2}\left(\begin{array}{ccc}
C_{0}(\eta) & 0 & 0 \\
0 & 1 & 0 \\
0 & 0 & 1
\end{array}\right)+\left(\begin{array}{ccc}
-\beta h(\eta) & 0 & 0 \\
0 & 0 & 0 \\
0 & 0 & 0
\end{array}\right) .
\end{aligned}
$$

Eq. (3) takes account of the low energy behaviour of $\mathbf{t}$ induced by the long range part of the Coulomb interaction. The quantities appearing in Eq. (3) are

$$
\begin{aligned}
& \mathbf{Q}=\left(\begin{array}{ccc}
q_{c} & 0 & 0 \\
0 & q_{0} & 0 \\
0 & 0 & k
\end{array}\right), \quad C_{0}(\eta)=\frac{2 \pi \eta}{\exp (2 \pi \eta)-1}, \\
& h(\eta)=-\ln |\eta|+\Re \psi(1+i \beta),
\end{aligned}
$$

with

$$
\beta=2 \alpha m_{c}, \quad \eta=-\alpha m_{c} / q_{c}, \quad m_{c}=\mu_{c} m_{p} /\left(\mu_{c}+m_{p}\right) .
$$

The matrix $\mathbf{K}$ is real-valued for $s>\left(\mu_{c}+m_{p}\right)^{2}$ and can be analytically continued into the $s$-plane.

The formalism of Ref. [6] will now be used to obtain the conversion formulae. Strictly speaking, the formalism relates $\Delta W$ and $\Gamma$ to $K_{c c}$ and $K_{c 0}$, evaluated at the position of the $1 s$ level. For reasons discussed in Section 3, phase shift analysis of low energy $\pi^{-} p$ elastic scattering does not give any reliable knowledge of the range parameters for $K_{c c}$ and $K_{c 0}$. However, it is sufficient to note that the differences between the values of $K_{c c}$ and $K_{c 0}$ at the $\pi^{-} p$ threshold and at the position of the $1 \mathrm{~s}$ level are expected to be of order $1 \times 10^{-6} \mathrm{fm}$, well below the uncertainties due to the experimental error. We therefore work from now on with the matrix a defined above. Following Ref. [6] we introduce the matrix $\mathbf{A}$ by

$$
\mathbf{A}^{-1}=\mathbf{a}^{-1}-i\left(\begin{array}{ccc}
0 & 0 & 0 \\
0 & q_{0} & 0 \\
0 & 0 & k
\end{array}\right)
$$

where $q_{0}$ and $k$ are from now on the c.m. channel momenta at the $\pi^{-} p$ threshold $q_{c}=0$. Numerically we will use $q_{0}=28.0408(96) \mathrm{MeV} / c$ and $k=129.40705(31) \mathrm{MeV} / c$.

From Eq. (5) we have

$$
\begin{aligned}
& a_{c c}=\Re A_{c c}+q_{0}^{2} a_{c 0}^{2} a_{00}+2 q_{0} k a_{c 0} a_{c \gamma} a_{0 \gamma}+k^{2} a_{c \gamma}^{2} a_{\gamma \gamma}, \\
& a_{c 0}=-\left|A_{c 0}\right|\left(1+q_{0} k a_{0 \gamma}^{2}+\frac{1}{2} q_{0}^{2} a_{00}^{2}-P^{-\frac{1}{2}} q_{0}^{\frac{1}{2}} k^{\frac{3}{2}} a_{0 \gamma} a_{\gamma \gamma}-\frac{1}{2} P^{-1} q_{0} k a_{0 \gamma}^{2}\right) .
\end{aligned}
$$

Eq. (6) is straightforward; Eq. (7) uses the result $a_{c \gamma}=-a_{c 0} q_{0}^{\frac{1}{2}} k^{-\frac{1}{2}} P^{-\frac{1}{2}}$, where $P$ is the Panofsky ratio, whose value [21] is 1.546(9). Eqs. (6), (7) give only the lowest terms in an 
expansion. To assess the magnitude of these terms we need estimates for $a_{c 0}, a_{c \gamma}, a_{00}, a_{0 \gamma}, a_{\gamma \gamma}$. The values of these quantities will be discussed in Section 3. Numerically, we find that

$$
a_{c c}=\Re A_{c c}\left(1+4.9 \times 10^{-5}\right), \quad a_{c 0}=-\left|A_{c 0}\right|\left(1+1.1 \times 10^{-6}\right) .
$$

The correction terms in Eq. (8) are negligible.

The expression for $\Delta W$ is given in Eq. (12) of Ref. [6] with $n=1, l=0$. Keeping only the numerically significant terms we have

$$
\frac{\Delta W}{W(1 s)^{\mathrm{pc}}}=\Re \epsilon_{0}\left(1+p_{1} \Re \epsilon_{0}\left[1-\left(\frac{\Im \epsilon_{0}}{\Re \epsilon_{0}}\right)^{2}\right]+p_{2}\left(\Re \epsilon_{0}\right)^{2}\right),
$$

where

$$
W(1 s)^{\mathrm{pc}}=-\frac{1}{2} \alpha^{2} m_{c}, \quad \Re \epsilon_{0}=4 a_{c c} / B, \quad \Im \epsilon_{0}=4 q_{0}\left(1+P^{-1}\right)\left|a_{c 0}\right|^{2} / B,
$$

$B=1 / \alpha m_{c}$ being the lowest Bohr radius of the $\pi^{-} p$ atom. The numerical values are $m_{c}=$ $121.49719(26) \mathrm{MeV}, W(1 s)^{\mathrm{pc}}=-3234.945(7) \mathrm{eV}$ and $B=222.56399(48) \mathrm{fm}$. The quantity $p_{1}=1 / 2(1+\gamma)=0.78860783, \gamma$ being Euler's constant. The expression for $p_{2}$ is given in Ref. [6]. The term involving a poorly known effective range parameter is small, and the result $p_{2}=-0.22$ is sufficiently accurate for our purpose.

From the experiment [3], Ref. [4] gives $W(3 p \rightarrow 1 s)=2885.928(10)$ eV. Using Eq. (2), this results in $\Delta W=-7.116(13) \mathrm{eV}$. With this number as the starting point for the iteration of Eq. (9), we obtain

$$
a_{c c}(\mathrm{fm})=0.0171704(1)(-\Delta W(\mathrm{eV})) .
$$

The error in the constant in Eq. (11) comes from the uncertainty in $m_{c}$ (due almost entirely to the error in $\mu_{c}$ ) and the uncertainty in $\Delta W$ (via the iteration). However, it is so small that it is of no consequence and will be disregarded in what follows. Using Eq. (2), the first conversion formula is

$$
a_{c c}(\mathrm{fm})=(0.0171704)[W(3 p \rightarrow 1 s)(\mathrm{eV})-2878.812(8)] .
$$

Numerically we obtain

$$
a_{c c}=0.12218(22) \mathrm{fm} \text {. }
$$

We would like to stress that the present experimental results for $W(3 p \rightarrow 1 s)$ and for $\Gamma[4,5]$ are consistent with, but more precise than, the earlier ones given in Ref. [2]. The result in Eq. (13) is by far the most accurate number in low energy $\pi N$ physics, and further refinement will not be useful, in view of the uncertainty in $\mu_{c}$ and even more of the uncertainty in the EM corrections to be discussed later.

We come now to the measured total width $\Gamma$ for the decay of the atomic $1 s$ state into both the $\pi^{0} n$ and $\gamma n$ channels. The numerical result for the Panofsky ratio $P$ quoted earlier is used first to obtain the partial width $\Gamma_{0}$ for decay into $\pi^{0} n$ :

$$
\Gamma=\left(1+P^{-1}\right) \Gamma_{0} .
$$

Vacuum polarisation and the finite extension of the charges each have a small but significant effect on $\Gamma_{0}$. The subthreshold calculation discussed earlier in connection with the shift shows that $V^{\text {ext }}$ changes $\Gamma_{0}$ by the factor 0.9948 , while $V^{\mathrm{vp}}$ changes it by the factor 1.0049 . The total effect of $\left(V^{\mathrm{vp}}+V^{\mathrm{ext}}\right)$ is a change by the factor 0.9996 . 
The treatment of decay widths on pages 410 and 411 of Ref. [6] gives

$$
\Gamma_{0}=4 \alpha^{3} m_{c}^{2} q_{0} a_{c 0}^{2}\left(1+2 p_{1} \Re \epsilon_{0}+p_{1}^{2}\left|\epsilon_{0}\right|^{2}+2 p_{2}\left(\Re \epsilon_{0}\right)^{2}\right),
$$

keeping only terms which are significant numerically and replacing $\left|A_{c 0}\right|^{2}$ by $a_{c 0}^{2}$ (see Eq. (8)). Combining Eqs. (14), (15) and using numerical values already given leads to the second conversion formula

$$
a_{c 0}(\mathrm{fm})=-0.19042(22)(\Gamma(\mathrm{eV}))^{1 / 2} .
$$

Almost all the error in Eq. (16) arises from the uncertainty in $P$. An updated result for $\Gamma$ from the experiment [3] is $0.823(19) \mathrm{eV}$ [5]. This leads via Eq. (16) to

$$
a_{c 0}=-0.1727(20) \mathrm{fm} \text {. }
$$

All the error in Eq. (17) comes from the experimental uncertainty on $\Gamma$; the error due to the uncertainty of the conversion constant is negligible. The experiment [3] finally hopes to achieve an accuracy of $1 \%$ (an absolute uncertainty of $0.008 \mathrm{eV}$ ), which will reduce the error on $a_{c 0}$ to about $0.0009 \mathrm{fm}$. Even in this case the error due to the conversion constant is only $2 \times 10^{-5} \mathrm{fm}$; therefore, a more accurate measurement of the Panofsky ratio would not improve the accuracy of the final value of $a_{c 0}$.

\section{Calculation of electromagnetic corrections}

To calculate the electromagnetic corrections to $a_{c c}$ and $a_{c 0}$ due to the point-charge Coulomb interaction, the external mass differences and the presence of the $\gamma n$ channel, we set up a potential model for the physical three-channel situation at the $\pi^{-} p$ threshold. The relativised Schrödinger equation (RSE) for the $s$-wave has the form

$$
\left[d^{2} / d r^{2} \mathbf{1}_{3}+\mathbf{Q}^{2}-2 \tilde{\mathbf{m}}\left(\tilde{\mathbf{V}}+\mathbf{V}^{\mathrm{pc}}\right)\right] \mathbf{u}(r)=\mathbf{0},
$$

where, since we are working at the $\pi^{-} p$ threshold $q_{c}=0$,

$$
\mathbf{Q}=\left(\begin{array}{ccc}
0 & 0 & 0 \\
0 & q_{0} & 0 \\
0 & 0 & k
\end{array}\right),
$$

with $q_{0}$ and $k$ having the same meaning as in Eq. (5). The diagonal matrix $\tilde{\mathbf{m}}$ of modified reduced masses is

$$
\tilde{\mathbf{m}}=\left(\begin{array}{ccc}
\tilde{m}_{c} & 0 & 0 \\
0 & \tilde{m}_{0} & 0 \\
0 & 0 & \tilde{m}_{\gamma}
\end{array}\right) .
$$

For a two-body channel with masses $m_{1}$ and $m_{2}$, the modified reduced mass is

$$
\tilde{m}=\frac{W^{2}-m_{1}^{2}-m_{2}^{2}}{2 W} \text {. }
$$

Thus, for $W=\mu_{c}+m_{p}$,

$$
\begin{aligned}
& \tilde{m}_{c}=\frac{\mu_{c} m_{p}}{\mu_{c}+m_{p}}=m_{c}, \quad \tilde{m}_{0}=\frac{\left(\mu_{c}+m_{p}\right)^{2}-\mu_{0}^{2}-m_{n}^{2}}{2\left(\mu_{c}+m_{p}\right)}, \\
& \tilde{m}_{\gamma}=\frac{\left(\mu_{c}+m_{p}\right)^{2}-m_{n}^{2}}{2\left(\mu_{c}+m_{p}\right)} .
\end{aligned}
$$


The reasons for the inclusion of these relativistic modifications of the reduced masses for the $\pi^{-} p$ and $\pi^{0} n$ channels were discussed in detail in Section 3 of Ref. [22], for the two-channel potential model calculation of the EM corrections for the analysis of low energy $\pi^{-} p$ scattering data. The modification is essential for the $\gamma n$ channel, where the standard reduced mass is zero, whereas $\tilde{m}_{\gamma}$ in Eq. (20) is in fact just $k$.

The $3 \times 3$ matrix $\mathbf{V}^{\mathrm{pc}}$ in Eq. (18) is

$$
\mathbf{V}^{\mathrm{pc}}=\left(\begin{array}{ccc}
V^{\mathrm{pc}} & 0 & 0 \\
0 & 0 & 0 \\
0 & 0 & 0
\end{array}\right),
$$

where $V^{\mathrm{pc}}$ is the attractive point-charge Coulomb potential. We recall that the effects of vacuum polarisation and of the finite extension of the charges of $\pi^{-}$and $p$ have already been accounted for. We write the matrix $\tilde{\mathbf{V}}$ in the form

$$
\tilde{\mathbf{V}}=\left(\begin{array}{ccc}
\tilde{V}_{c c} & \tilde{V}_{c 0} & V_{c \gamma} \\
\tilde{V}_{c 0} & \tilde{V}_{00} & V_{0 \gamma} \\
V_{c \gamma} & V_{0 \gamma} & V_{\gamma \gamma}
\end{array}\right) \quad \text { and define the submatrix } \tilde{\mathbf{V}}_{h}=\left(\begin{array}{cc}
\tilde{V}_{c c} & \tilde{V}_{c 0} \\
\tilde{V}_{c 0} & \tilde{V}_{00}
\end{array}\right) .
$$

We shall call $\tilde{\mathbf{V}}_{h}$ the EM modified hadronic potential because, as explained in Section 1, it contains residual EM effects. There is clear evidence, to be discussed in Section 5, that $\tilde{\mathbf{V}}_{h}$ violates isospin invariance, though only by a few percent. However, we have only the numbers $a_{c c}$ and $a_{c 0}$ available experimentally ( $a_{00}$ is inaccessible) and therefore need to express $\tilde{\mathbf{V}}_{h}$ in terms of two parameters. Hence we made the approximation of treating $\tilde{\mathbf{V}}_{h}$ as isospin invariant:

$$
\tilde{\mathbf{V}}_{h}=\left(\begin{array}{cc}
\frac{2}{3} \tilde{V}_{1 / 2}+\frac{1}{3} \tilde{V}_{3 / 2} & \frac{\sqrt{2}}{3}\left(\tilde{V}_{3 / 2}-\tilde{V}_{1 / 2}\right) \\
\frac{\sqrt{2}}{3}\left(\tilde{V}_{3 / 2}-\tilde{V}_{1 / 2}\right) & \frac{1}{3} \tilde{V}_{1 / 2}+\frac{2}{3} \tilde{V}_{3 / 2}
\end{array}\right) .
$$

We made the same approximation in Ref. [22] in order to calculate EM corrections for the analysis of low energy $\pi^{-} p$ scattering data. Since this potential is used solely for the calculation of the corrections, this approximation should have very little effect on the results. For $\tilde{V}_{1 / 2}$ and $\tilde{V}_{3 / 2}$ we assumed the radial dependence of the $s$-wave hadronic potentials used in Refs. [10,22] and left their strengths as parameters to be adjusted. The leading term in each potential is of Gaussian form, with a range parameter which is fixed at $1 \mathrm{fm}$ (a realistic value for the hadronic interaction), and two small correction terms are added.

The three potentials $V_{c \gamma}, V_{0 \gamma}$ and $V_{\gamma \gamma}$ in Eq. (21) also need to have some assumed radial dependence, with their strengths left as parameters to be adjusted. A study of the analytic structure of the relevant amplitudes shows that the range parameter $1 \mathrm{fm}$ is also appropriate for these three potentials. For convenience we took the radial dependence of $V_{c \gamma}$ to be the same as that of $\tilde{V}_{c 0}$, and the radial dependence of $V_{0 \gamma}$ and $V_{\gamma \gamma}$ to be the same as that of $\tilde{V}_{00}$. In the calculation there were then five potential strengths to be adjusted, with five experimentally determined numbers used to fix them.

In addition to the values of $a_{c c}$ and $a_{c 0}$ given in Eqs. (13), (17), we used experimentally determined values of $a_{c \gamma}, a_{0 \gamma}$ and $a_{\gamma \gamma}$. From the values of $a_{c 0}$ and $P$, we obtain $a_{c \gamma}=0.0647 \mathrm{fm}$. The value of $a_{0 \gamma}$ can be obtained only from an analysis of experimental data near threshold for the photoproduction process $\gamma n \rightarrow \pi^{0} n$. The results given in Ref. [23] lead to $a_{0 \gamma}=-0.0028 \mathrm{fm}$, but no error is given; we think that $0.0006 \mathrm{fm}$ is a realistic uncertainty. The value of $a_{\gamma \gamma}$ comes from the analysis of data on Compton scattering off neutrons. From results in Ref. [24] we have extracted $a_{\gamma \gamma}=0.0011 \mathrm{fm}$, with an uncertainty of around $20 \%$. The five potential strengths 
were then varied until they reproduced the five numbers just discussed, thus fixing $\tilde{\mathbf{V}}$ for the three-channel calculation.

We now give some details of the calculation of a from the RSE (18). For the two-channel case (omitting the $\gamma n$ channel) the calculation is given fully in Section 3 of Ref. [25]; it can easily be generalised to the three-channel situation. The first step is to form the matrix $\mathbf{P}(r)$ by putting side by side, as its three columns, three linearly independent regular solutions $\mathbf{u}^{j}(r), j=1,2,3$, of Eq. (18). Let $R$ be a distance beyond which $\tilde{\mathbf{V}}$ is negligible. We then define the real symmetric matrix $\mathbf{d}$ by

$$
\mathbf{d}=R \tilde{\mathbf{m}}^{-1 / 2} \mathbf{P}^{\prime}(R)[\mathbf{P}(R)]^{-1} \tilde{\mathbf{m}}^{1 / 2},
$$

where the prime denotes differentiation with respect to $r$ and the matrix elements of $\tilde{\mathbf{m}}$ are given in Eq. (20). Note that our present notation is different from that of Ref. [25]. The matrix $\mathbf{K}$ in Eq. (64) of that reference is now $\mathbf{t}$, defined just before Eq. (3), and $\mathbf{A}$ in Eq. (65) is now $\mathbf{K}$ as defined in Eq. (3). Eq. (66) of Ref. [25], generalised to the three-channel case and taken at threshold, gives

$$
\begin{aligned}
R^{-1} \mathbf{a}= & \left(\begin{array}{ccc}
\Phi_{0}^{*}-d_{c c} \Phi_{0} & -d_{c 0} \Phi_{0} & -d_{c \gamma} \Phi_{0} \\
-d_{c 0} \rho_{0}^{-1} \sin \rho_{0} & \cos \rho_{0}-d_{00} \rho_{0}^{-1} \sin \rho_{0} & -d_{0 \gamma} \rho_{0}^{-1} \sin \rho_{0} \\
-d_{c \gamma} \rho_{\gamma}^{-1} \sin \rho_{\gamma} & -d_{0 \gamma} \rho_{\gamma}^{-1} \sin \rho_{\gamma} & \cos \rho_{\gamma}-d_{\gamma \gamma} \rho_{\gamma}^{-1} \sin \rho_{\gamma}
\end{array}\right) \\
& \times\left(\begin{array}{ccc}
X_{0}^{*}+d_{c c} X_{0} & d_{c 0} X_{0} & d_{c \gamma} X_{0} \\
d_{c 0} \cos \rho_{0} & \rho_{0} \sin \rho_{0}+d_{00} \cos \rho_{0} & d_{0 \gamma} \cos \rho_{0} \\
d_{c \gamma} \cos \rho_{\gamma} & d_{0 \gamma} \cos \rho_{\gamma} & \rho_{\gamma} \sin \rho_{\gamma}+d_{\gamma \gamma} \cos \rho_{\gamma}
\end{array}\right)^{-1},
\end{aligned}
$$

where $\rho_{0}=q_{0} R$ and $\rho_{\gamma}=k R$. The auxiliary Coulomb functions $\Phi_{0}, \Phi_{0}^{*}, X_{0}$ and $X_{0}^{*}$ (discussed at length in Section 2 of Ref. [25]) have at threshold the arguments $(-\beta R ; 0)$, which we have suppressed. In terms of Bessel functions they are, with $x=2 \sqrt{\beta R}$,

$$
\Phi_{0}=2 x^{-1} J_{1}(x), \quad \Phi_{0}^{*}=J_{0}(x), \quad X_{0}=-\frac{1}{2} \pi x Y_{1}(x), \quad X_{0}^{*}=\frac{1}{4} x^{2} Y_{0}(x) .
$$

Having given the details of the modelling of the three-channel situation, we now proceed to calculate the corrections which remove from $a_{c c}$ and $a_{c 0}$ the effects of the point charge Coulomb potential, of the mass differences $\mu_{c}-\mu_{0}$ and $m_{n}-m_{p}$, and of the presence of the $\gamma n$ channel. To obtain them, we need to model the two-channel situation with these three effects removed, using the RSE at threshold

$$
\left[d^{2} / d r^{2} \mathbf{1}_{2}-2 m_{c} \tilde{\mathbf{V}}_{h}\right] \mathbf{u}(r)=\mathbf{0} .
$$

The appearance of $m_{c}$ in Eq. (24) implies the assignment of the mass $\mu_{c}$ to both $\pi^{-}$and $\pi^{0}$, and the mass $m_{p}$ to both $p$ and $n$. This choice is made in most studies of the low energy $\pi N$ system and was discussed fully in Section 5.2 of Ref. [9]. Thus the scattering parameters $\tilde{a}_{c c}$ and $\tilde{a}_{c 0}$ which result from Eq. (24) still contain residual EM contributions.

By integrating Eq. (24) we obtain the $2 \times 2$ matrix $\tilde{\mathbf{a}}$; the numerical values for the relevant matrix elements are

$$
\tilde{a}_{c c}=0.12140 \mathrm{fm}, \quad \tilde{a}_{c 0}=-0.1757 \mathrm{fm} .
$$

We now define the stage 1 EM corrections

$$
\Delta^{1} a_{c c}=a_{c c}-\tilde{a}_{c c}, \quad \Delta^{1} a_{c 0}=a_{c 0}-\tilde{a}_{c 0} .
$$


From Eqs. (13), (17), (25) and (26), we have

$$
\Delta^{1} a_{c c}=+0.00078 \mathrm{fm}, \quad \Delta^{1} a_{c 0}=+0.0030 \mathrm{fm} .
$$

These are the essential results from the present calculation.

It is instructive to look at the contributions of the three effects to the corrections $\Delta^{1} a_{c c}$ and $\Delta^{1} a_{c 0}$. For $\Delta^{1} a_{c c}$ we have

$$
\begin{aligned}
& \Delta a_{c c}(p c)=-0.00214 \mathrm{fm}, \quad \Delta a_{c c}(m d)=-0.00071 \mathrm{fm}, \\
& \Delta a_{c c}(\gamma n)=+0.00363 \mathrm{fm} .
\end{aligned}
$$

The smallness of the total correction is thus seen to be the result of a near cancellation between the effect of the $\gamma n$ channel, which is large, and the combined effect of $V^{\mathrm{pc}}$ and the mass differences. One sees how important it is to estimate the effect of the $\gamma n$ channel as reliably as possible. For $\Delta^{1} a_{c 0}$ the situation is quite different. We now have

$$
\begin{aligned}
& \Delta a_{c 0}(p c)=-0.0002 \mathrm{fm}, \quad \Delta a_{c 0}(m d)=+0.0029 \mathrm{fm}, \\
& \Delta a_{c 0}(\gamma n)=+0.0003 \mathrm{fm} .
\end{aligned}
$$

The effect of the $\gamma n$ channel is now very small, and the correction is due almost entirely to the mass differences.

Uncertainties in the corrections given in Eq. (27) arise from: (a) the shapes assumed for the components of $\tilde{\mathbf{V}}_{h}$; (b) the errors of the five experimentally determined quantities which are fitted; (c) the approximation of using an isospin invariant form for $\tilde{\mathbf{V}}_{h}$. The quantity $\Delta a_{c c}(p c)$ has a significant uncertainty due to lack of knowledge of the shapes of the components of $\tilde{\mathbf{V}}_{h}$. This was estimated as in Refs. [10,22] by varying the range parameter between $0.8 \mathrm{fm}$ and $1.2 \mathrm{fm}$ and simultaneously adjusting the strengths of the potentials so that the five scattering parameters discussed earlier in this section remain constant. The quantity $\Delta a_{c c}(\gamma n)$ suffers from the same uncertainty, and has in addition a significant error due to the uncertainty in $a_{c \gamma}, a_{0 \gamma}$ and $a_{\gamma \gamma}$. The uncertainty in $\Delta a_{c 0}(m d)$, the dominant part of $\Delta^{1} a_{c 0}$, comes from uncertainties in $\tilde{\mathbf{V}}_{h}$ (the shapes of $\tilde{V}_{1 / 2}$ and $\tilde{V}_{3 / 2}$ and the assumption of isospin invariance).

Unfortunately, in the situation under consideration one cannot achieve the precision associated with the errors quoted in the experimental results. The variations described in the previous paragraph can lead to rather rough estimates only. It is therefore wise to err on the side of caution and increase these estimates accordingly. We think that, on the basis of the estimates we have made, the errors on each of the components of the corrections could be as high as $20 \%$. This leads to the following final values for the corrections

$$
\Delta^{1} a_{c c}=+0.0008(8) \mathrm{fm}, \quad \Delta^{1} a_{c 0}=+0.0030(6) \mathrm{fm} .
$$

Combining Eqs. (13), (17) and (30), we obtain the final results

$$
\tilde{a}_{c c}=0.1214(8) \mathrm{fm}, \quad \tilde{a}_{c 0}=-0.1757(21) \mathrm{fm} .
$$

(We have given results for the $s$-wave scattering parameters in fm because it is a precisely defined unit. In units which are in common use, the final results in Eq. (31) are $\tilde{a}_{c c}=0.0859(6) \mu_{c}^{-1}$ and $\tilde{a}_{c 0}=-0.1243(15) \mu_{c}^{-1}$.)

It is important to note that the error in $\tilde{a}_{c c}$ arises almost entirely from the uncertainty in $\Delta^{1} a_{c c}$, so that an improvement in the accuracy of the measurement of $W(3 p \rightarrow 1 s)$ will not reduce the error in $\tilde{a}_{c c}$. On the other hand, almost all the error in $\tilde{a}_{c 0}$ comes from the experimental 
uncertainty in $\Gamma$. If the error in the measurement of $\Gamma$ can be reduced to $1 \%$, the uncertainty in $\tilde{a}_{c 0}$ in Eq. (31) will be reduced from (21) to (11).

In the discussion following Eq. (35) of Ref. [9], we emphasised the very large difference between the values of $\tilde{a}_{c c}$ which come from pionic $\mathrm{H}$ and from our PSA of the combined $\pi^{+} p$ and $\pi^{-} p$ elastic scattering databases for $T_{\pi} \leqslant 100 \mathrm{MeV}$. Converting from the unit $\mu_{c}^{-1}$ to fm, the latter gives the value $\tilde{a}_{c c}=0.1127(16) \mathrm{fm}$. The difference between this value and the value $0.1214(8) \mathrm{fm}$ given in Eq. (31) is thus 0.0087(18) fm. This large difference is a subtle consequence of the violation of isospin invariance in the electromagnetically modified hadronic interaction, which is convincingly demonstrated in Section 7 of Ref. [9] and in Section 5 of this paper. The phase shift $\tilde{\delta}_{1 / 2}$ used in Ref. [9] to analyse $\pi^{-} p$ elastic scattering data is an artificial construct, which enables the analysis to be done in a framework of formal isospin invariance. When isospin invariance is violated, $\tilde{\delta}_{1 / 2}$ goes to a nonzero value at threshold, so that the scattering length $\tilde{a}_{1 / 2}$ given in Ref. [9] is an artefact of the parameterisation of $\tilde{\delta}_{1 / 2}$, which forces it to behave like $q_{c}$ near threshold, and the extrapolation to threshold (using this artificial parameterisation) of values of $\tilde{\delta}_{1 / 2}$ obtained from data at momenta $q_{c} \approx 80 \mathrm{MeV} / c$ upwards. Therefore the value of $\tilde{a}_{c c}$ extracted from pionic $\mathrm{H}$ cannot be compared directly with the value of $\left(2 \tilde{a}_{1 / 2}+\tilde{a}_{+}\right) / 3$ obtained in the PSA of Ref. [9].

\section{Comparison with other calculations of the electromagnetic corrections}

As mentioned already in the introduction, in our method of calculating the EM corrections to the pionic $\mathrm{H}$ data we use hadronic potentials which are of the same form as those of Refs. [10, 22]. This implies that the EM corrections which were applied to the low energy $\pi^{ \pm} p$ scattering data in our PSA of Ref. [9] are compatible with the ones obtained in the present paper for the pionic $\mathrm{H}$ case. For the PSA, it was sufficient, in analysing data from $q_{c} \approx 80 \mathrm{MeV} / c$ upwards, to take the $\gamma n$ channel into account by using the perturbative result of Ref. [26]. It has practically no effect on the results of the PSA. However, for our calculation at threshold, the effect of the $\gamma n$ channel turns out to be very significant in the case of the correction to $a_{c c}$, thus justifying the full three-channel calculation we have made. In these respects, our calculations of the EM corrections to the pionic $\mathrm{H}$ data differ considerably from the older work of Refs. [16,27], both of which use primitive forms of the hadronic potentials which reproduce only the scattering lengths, in contrast to our forms which also reproduce the phase shifts for $T_{\pi} \leqslant 100 \mathrm{MeV}$. Furthermore, Ref. [27] is a non-relativistic calculation which uses much older values of the scattering lengths. In Ref. [16] the $\gamma n$ channel is taken into account in an unsatisfactory way, by using the Panofsky ratio to alter the input value of $a_{c 0}$ in what remains a two-channel calculation, whereas we have included this channel in the most realistic way possible in a three-channel calculation. In addition, Refs. [16,27] do not take account of the accurate relations given in Ref. [6], between $\Delta W$ and $a_{c c}$ and between $\Gamma_{0}$ and $a_{c 0}$. This means that our corrections and those of Ref. [16] differ by the factors in the parentheses of Eqs. (9), (15). For these reasons, we consider that our calculations supersede the earlier potential model calculations in Refs. [16,27].

A quite different method of calculating the EM corrections is used in Ref. [13]. In a nonrelativistic approach using Coulomb wave functions, with a short-range hadronic interaction and extended charge distributions of $\pi^{-}$and $p$, the authors identify four components of the corrections. Two are the contributions from the interference of $V^{\mathrm{vp}}$ and $V^{\text {ext }}$ with the hadronic potential $\tilde{V}$; the other two are a renormalisation term and a gauge term. The interference terms are obtained using an argument based on the change at the origin in the non-relativistic $1 s$ wave function arising from $V^{\mathrm{pc}}$, when $V^{\mathrm{vp}}$ and $V^{\mathrm{ext}}$ are added to $V^{\mathrm{pc}}$. This is a purely EM calculation, 
and the results are therefore claimed to be independent of $\tilde{V}$. We can see no theoretical justification for this procedure of artificially introducing the wave function at the origin as a replacement for the lowest Bohr energy in the DGBT formulae, and then calculating corrections via changes in this wave function. Moreover, while the shift in the $1 s$ level position due to the interference of $V^{\mathrm{vp}}$ with $\tilde{V}$ happens to be correct $(-0.0034 \mathrm{eV})$, that for $V^{\mathrm{ext}}$ comes out to be $+0.0060 \mathrm{eV}$, whereas the correct result is $+0.0034 \mathrm{eV}$, which gives the cancellation reported in Section 2 and in Ref. [16]. The subthreshold calculation discussed in Section 2 shows that these contributions separately have a significant variation with the range of the hadronic potential, and we have given the error which results from this for the sum of the contributions. Ref. [13] also incorrectly says that the results given there for the interference contributions agree with those given in Ref. [16].

While Ref. [13] has a discussion of the multichannel situation in Section 2.2, it does not explicitly give the terms which appear in parentheses in our Eqs. (9), (15), nor are these (admittedly small) terms taken into account in their corrections. Further, we cannot see any corrections in Ref. [13] which correspond to the corrections due to external mass differences appearing in Eqs. (28), (29).

That leaves the renormalisation and gauge terms in Ref. [13]. Each of these terms involves an integral over the extended charge distributions, so it appears that some of the effect of extended charges is contained in these terms. In our approach, however, $V^{\text {ext }}$ is fully accounted for by its direct contribution to the position of the $1 s$ level and the interference of $V^{\text {ext }}$ with $\tilde{V}$. It also does not seem possible to see where the interference of $V^{\mathrm{pc}}$ with $\tilde{V}$, which gives the corrections designated (pc) in Eqs. (28), (29), appears in the corrections of Ref. [13]. So here we deal with two very different approaches to the calculation of the EM corrections, with no discernible way of comparing them.

Further, the gauge terms in Ref. [13], for the corrections to $a_{c c}$ and $a_{c 0}$, are much more uncertain than is claimed there. They are proportional to the range parameters $b_{c c}^{h}$ and $b_{c 0}^{h}$ respectively, which appear in the low energy expansions of $K_{c c}$ and $K_{c 0}$. Ref. [13] uses for these quantities values given in Ref. [28], taken from a PSA of $\pi N$ scattering data obtained before 1983. Practically none of these data were used in our recent PSA [9]. The numbers in Ref. [28] are no longer of any value. Moreover, as we explained at the end of Section 3, because of the violation of isospin invariance in the electromagnetically modified hadronic interaction, the PSA of Ref. [9] does not lead to a reliable estimate of $\tilde{a}_{c c}$. For $\tilde{b}_{c c}$ the situation is even more uncertain. The value obtained from the PSA cannot be accepted as a reliable determination; it is merely a rough guess based on phase shifts for $q_{c} \gtrsim 80 \mathrm{MeV} / c$. The same holds for $\tilde{b}_{c 0}$. It is not possible at present to obtain reliable values of the range parameters from a PSA. As a result, the values of the EM corrections proposed in Ref. [13] are quite uncertain.

In our calculations, the range parameters are replaced by potentials which, as explained in Refs. [10,22], are constructed to reproduce the phase shifts for $q_{c} \gtrsim 80 \mathrm{MeV} / c$. The lack of experimental data at very low energies increases the uncertainty in the potentials; it allows a wider interval of values of the Gaussian range parameter $a$ which yield acceptable fits than would otherwise be the case. We have taken this uncertainty in $a$ into account in the estimates of the errors in our corrections given in Eq. (30).

In Ref. [29] the dispersive EM correction to $a_{c c}$ which arises from the radiative processes $\pi^{-} p \rightarrow \gamma X(X=n, \Delta)$ is calculated in the heavy baryon limit. Table 1 of Ref. [29] gives the correction $\Delta a_{c c}(\gamma n)=0.00424(13)$, while our result (Eq. (28)) is 0.00363(72) fm. These two results, which have been obtained in completely different ways, agree quite well. The case $X=\Delta$ is very different. In the potential model, the effect of a $\gamma \Delta$ intermediate state is a residual EM contribution to $\tilde{V}$, and has to be calculated in another way. The result given in Table 1 of Ref. [29] 
is $\Delta a_{c c}(\gamma \Delta)=+0.00332 \mathrm{fm}$. There is no other estimate of this correction. One should note that diagrams with a $\gamma \pi N$ intermediate state in principle appear in the calculation of Ref. [30], which we shall discuss in a moment, but at a higher order in the perturbation expansion. An effect due to $\gamma \Delta$ as large as that given in Ref. [29] would imply that higher orders of the expansion give contributions which are comparable in magnitude to the one-loop result given in Ref. [30].

Gasser et al. [30] have made a calculation of the EM correction to $a_{c c}$ using baryon chiral perturbation theory (ChPT) in the infrared regularisation. The calculation was carried out up to diagrams containing one loop; it is a calculation of the complete EM correction to $a_{c c}$ to this order. An unambiguous splitting of this correction into stage 1 and stage 2 components is a very difficult task, and may not even be possible (for example, due to the ultraviolet divergences). Nevertheless we can say that some parts of the correction correspond to the effects calculated (to all orders) with our potential model. In addition, there are much more important parts of the correction of Ref. [30], which are not taken into account in our calculation. They give a large negative correction which swamps the small correction $\Delta^{1} a_{c c}$ in Eq. (30). Unfortunately, this correction is very uncertain, due to a lack of knowledge of the low energy constant $f_{1}$.

For the width, there is a calculation using baryon ChPT reported in Ref. [31], but only to lowest order (no loops). It contains contributions which are not accounted for in the potential model calculation; the total contribution is positive, but very small compared with the correction $\Delta^{1} a_{c 0}$ in Eq. (30).

\section{Violation of isospin invariance}

If the electromagnetically modified hadronic interaction were to satisfy isospin invariance, we would have the equality

$$
\tilde{a}_{c c}+\sqrt{2} \tilde{a}_{c 0}=\tilde{a}_{+}
$$

where $\tilde{a}_{+}$is the $s$-wave scattering length for elastic $\pi^{+} p$ scattering. The value of $\tilde{a}_{+}$needs to be determined by means of a PSA of low energy $\pi^{+} p$ scattering data, using EM corrections calculated in a manner consistent with the calculation of the corrections in Eq. (30). Our analysis [9] of the $\pi^{+} p$ scattering data for $T_{\pi} \leqslant 100 \mathrm{MeV}$ gives

$$
\tilde{a}_{+}=-0.1062(55) \mathrm{fm} .
$$

When the analysis is made of a database which combines $\pi^{+} p$ and $\pi^{-} p$ elastic scattering data up to $100 \mathrm{MeV}$, using a more restrictive model for the fitting, the value of $\tilde{a}_{+}$is practically unaltered, but the error decreases from (55) to (23). For the purpose of testing for isospin invariance, the larger uncertainty in Eq. (32) should be used.

From the results in Eq. (31) we have

$$
\tilde{a}_{c c}+\sqrt{2} \tilde{a}_{c 0}=-0.1271(31) \mathrm{fm} .
$$

Almost all the error in Eq. (33) arises from the uncertainty in $\tilde{a}_{c 0}$. Reducing the error in $\Gamma$ to $1 \%$ would reduce the uncertainty in Eq. (33) to (17). The difference between the numbers in Eqs. (32), (33) is 0.0209(63) fm, which amounts to 3.3 standard deviations. This is evidence for a significant violation of isospin invariance at the electromagnetically modified hadronic level. If the experiment [3] achieves its hoped for accuracy for the measurement of $\Gamma$, the error in the difference will change from (63) to (58). If the result for $\Gamma$ remains approximately the same, the difference will become even more significant. 
This result strengthens the evidence for the violation of isospin invariance at the electromagnetically modified hadronic level given in Ref. [9], which comes from the inability of the phase shifts obtained from the analysis of the combined $\pi^{ \pm} p$ elastic scattering data to reproduce the data on the charge exchange reaction $\pi^{-} p \rightarrow \pi^{0} n$.

\section{Acknowledgements}

We are grateful to Detlev Gotta for communicating to us the current results of the PSI pionic hydrogen experiment, and to Jürg Gasser and Akaki Rusetsky for their helpful answers to our questions concerning the work of the Bern group. We would also like to thank the two reviewers of our paper for their useful recommendations and constructive criticism.

\section{References}

[1] S. Deser, M.L. Goldberger, K. Baumann, W. Thirring, Phys. Rev. 96 (1954) 774.

[2] H.-Ch. Schröder, et al., Eur. Phys. J. C 21 (2001) 473.

[3] PSI experiment R-98.01, http://pihydrogen.web.psi.ch.

[4] M. Hennebach, PhD Dissertation, Universität zu Köln, 2003.

[5] D. Gotta, private communication.

[6] G. Rasche, W.S. Woolcock, Nucl. Phys. A 381 (1982) 405.

[7] T.L. Trueman, Nucl. Phys. 26 (1961) 57.

[8] E. Lambert, Helv. Phys. Acta 42 (1969) 667.

[9] E. Matsinos, W.S. Woolcock, G.C. Oades, G. Rasche, A. Gashi, Nucl. Phys. A 778 (2006) 95.

[10] A. Gashi, E. Matsinos, G.C. Oades, G. Rasche, W.S. Woolcock, Nucl. Phys. A 686 (2001) 447.

[11] D. Chatellard, et al., Nucl. Phys. A 625 (1997) 855.

[12] T.E.O. Ericson, B. Loiseau, A.W. Thomas, Phys. Rev. C 66 (2002) 014005.

[13] T.E.O. Ericson, B. Loiseau, S. Wycech, Phys. Lett. B 594 (2004) 76.

[14] U.-G. Meißner, U. Raha, A. Rusetsky, Phys. Lett. B 639 (2006) 478.

[15] W.-M. Yao, et al., J. Phys. G 33 (2006) 1.

[16] D. Sigg, A. Badertscher, P.F.A. Goudsmit, H.J. Leisi, G.C. Oades, Nucl. Phys. A 609 (1996) 310.

[17] G.J.M. Austen, J.J. de Swart, Phys. Rev. Lett. 50 (1983) 2039.

[18] H. de Vries, C.W. de Jager, C. de Vries, At. Data Nucl. Data Tables 36 (1987) 495.

[19] S.R. Amendolia, et al., Nucl. Phys. B 277 (1986) 168.

[20] W.S. Woolcock, G. Rasche, Cross sections and decay rates, in: C.J. Burden, B.A. Robson (Eds.), Nuclear and Particle Physics, World Scientific, Singapore, 1990.

[21] J. Spuller, et al., Phys. Lett. B 67 (1977) 479;

See also T. Flügel, PhD Dissertation, Swiss Federal Institute of Technology, Zürich, 1999.

[22] A. Gashi, E. Matsinos, G.C. Oades, G. Rasche, W.S. Woolcock, Nucl. Phys. A 686 (2001) 463.

[23] R.A. Arndt, W.J. Briscoe, I.I. Strakovsky, R.L. Workman, Phys. Rev. C 66 (2002) 055213.

[24] A.I. L’vov, Sov. J. Nucl. Phys. 42 (1985) 583.

[25] G. Rasche, W.S. Woolcock, Helv. Phys. Acta 49 (1976) 455.

[26] B. Tromborg, S. Waldenstrøm, I. Øverbø, Phys. Rev. D 15 (1977) 725;

B. Tromborg, S. Waldenstrøm, I. Øverbø, Helv. Phys. Acta 51 (1978) 584.

[27] W.B. Kaufmann, W.R. Gibbs, Phys. Rev. C 35 (1987) 838.

[28] G. Höhler, Pion-nucleon scattering, in: H. Schopper (Ed.), Landolt-Börnstein, vol. I/9b2, Springer, Berlin, 1983.

[29] T.E.O. Ericson, A.N. Ivanov, Phys. Lett. B 634 (2006) 39.

[30] J. Gasser, M.A. Ivanov, E. Lipartia, M. Mojžiš, A. Rusetsky, Eur. Phys. J. C 26 (2002) 13.

[31] P. Zemp, PhD Dissertation, University of Bern, 2004. 